\title{
Projeto Bandeira Científica: história, estratégias e resultados
}

\author{
Luiz Fernando Ferraz da Silva
}

Silva LFF. Projeto Bandeira Científica: história, estratégias e resultados. Rev Med (São Paulo). 2012 jan.-mar.;91(1):36-43.

RESUMO: Projeto Bandeira Científica é um projeto de extensão criado em 1957 que atualmente tem caráter interdisciplinar e que desenvolve anualmente atividades educacionais, científicas e assistenciais em diferentes municípios do país. O projeto conta com uma equipe anual média de 160 alunos e 50 profissionais atuando de forma interdisciplinar e conta com uma fase de preparação de 8 meses para levantamento de demandas e preparação da equipe, uma expedição de 10 dias para atividades assistenciais, educativas e de coleta de dados e atividades de seguimento presencial e à distância por outros 8 meses incluindo a proposição de projetos estruturantes em saúde. Nos últimos 14 anos já atingiu diretamente mais de 45.000 pessoas em 17 municípios do país e contou com a participação de 1428 alunos de graduação que tiveram oportunidade de vivenciar uma realidade completamente diferente do habitual que representa a realidade de grande parte do país. Parcerias com 22 instituições até o momento possibilitaram ampliação do espectro de continuidade e replicação do projeto. Dados científicos levantados durante a expedição mostram ótimo nível de produtividade no contexto de um projeto de extensão. Embora a repercussão positiva do projeto seja considerável, entendemos que sempre há espaço para aprimoramento, especialmente no que tange às estratégias de atuação interdisciplinar e continuidade.

DESCRITORES: Assistência à saúde/recursos humanos; Expedições/história; Relações comunidade-instituição; Atenção à saúde.

\begin{abstract}
The "Bandeira Cientifica" is an universitary extension project created in 1957 that annually develops interdisciplinary educational, scientific and health care activities in different cities of Brazil. The project team includes 160 students and 50 professionals working in an interdisciplinary way. It includes (1) a preparatory phase ( 8 months) to identify cities demands as well as to select and prepare the students and professionals that will take part on the (2) expedition (lasting 10 days) in which they develop health care, scientific data collection and educational activities. These data are the basis of (3) follow-up activities (8 months) with seen face and on distance activities and meetings including the proposition of structural projects to guide public policies. Over the last 14 years "Bandeira Científica" has reached directly more than 45,000 people in 17 municipalities of the country and did count on 1428 undergraduate students who have had the opportunity to experience a completely different reality (than their usual), that represents the reality of much of the country. Partnerships with 22 institutions so far allowed broadening the continuity and replication of the project. Scientific results show optimum level of productivity in the context of an extension project. Although the fact that the positive impact of the project is considerable, we believe that there is always possibilities for improvement, especially in strategies for interdisciplinary action and continuity.
\end{abstract}

KEYWORDS: Delivery of health care/manpower; Expedition, Expeditions/history; Community-instiutional relations Health care (Public health).

Diretoria do Projeto Bandeira Científica. Faculdade de Medicina da USP - Departamento de Patologia.

Endereço para correspondência: Av. Dr. Arnaldo, 455 - 1ํandar - Sala 1155 - São Paulo, SP. CEP: 01246-903. e-mail: burns @ usp. $\mathrm{br}$ 


\section{INTRODUÇÃO E ASPECTOS HISTÓRICOS}

\begin{abstract}
$\Delta$ Bandeira Científica é um projeto acadêmico de extensão universitária organizado por acadêmicos de múltiplas unidades da Universidade de São Paulo sob a supervisão de docentes destas mesmas unidades que tem por objetivo desenvolver atividades interdisciplinares focadas em saúde em municípios menos favorecidos e/ou com particularidades na atenção à saúde, ao mesmo tempo que proporciona ao graduando a experiência de vivenciar uma realidade diferenciada e a aplicação de seus conhecimentos técnicos com as limitações inerentes a estas realidades e o estímulo à atuação interdisciplinar.

A cada ano, a Bandeira Científica vem buscando atuar de forma a garantir o desenvolvimento sustentável de saúde da região, saúde aqui entendida como o "completo estado de bem-estar bio-psicosocial, não apenas a ausência de doença". Este é um conceito e objetivo bastante atual, mas que passou por um longo processo de amadurecimento cuja compreensão pode ser obtida ao se analisar as fases históricas do projeto iniciadas em meados do século passado.
\end{abstract}

\section{Fase 1 - Da criação à interrupção abrupta}

A Bandeira Científica foi criada em 1957

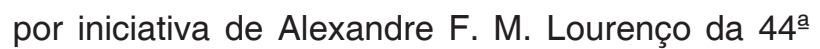
Turma de medicina da Faculdade de Medicina da USP (FMUSP) que com o apoio de outros alunos da casa idealizou uma expedição ao pantanal do Mato Grosso para a realização de atividades de pesquisa sob a coordenação do Prof. Luis Rey. Esta primeira expedição partiu no dia 1ำ de janeiro de 1958 onde por cerca de um mês a equipe cobriu quatro cidades da região coletando informações ${ }^{2}$. A adesão foi tamanha que em 1959 uma nova expedição saía desta vez para Pernambuco ${ }^{3}$. Seguiram-se mais nove expedições em municípios do Ceará (Vale do Cariri, Sobral e Viçosa), Pará (Vila de Santana), Amapá (Macapá, Vila de Santana e Serra do Navio), Pará - Ilha de Marajó (Cachoeira do Arari), Bahia (Ilhéus e Uruçuca) e Rio Grande do Sul (Torres) - incluir foto. Em 1969 as Bandeiras foram interrompidas devido à realidade político-social da época incluindo a aposentadoria compulsória do Prof. Luis Rey e Luiz Hildebrando Pereira da Silva - grandes entusiastas e apoiadores do projeto

\section{Fase 2 - A refundação}

Após uma latência de quase 30 anos, em 1997 um grupo de alunos comandados pelo acadêmico
Rafael Bernardon Ribeiro, da $85^{\mathrm{a}}$ turma da FMUSP que consultava arquivos da Faculdade de Medicina da USP se mobilizou para reativá-la. As ações foram retomadas em 1998, sob coordenação do Prof. Paulo Hilário Nascimento Saldiva do departamento de patologia, passando à configuração de Projeto de Extensão Universitária da USP em 2000. Além das atividades fundamentais de educação e pesquisa (herdadas do conceito inicial da Bandeira), a partir de 1999 foi introduzida a vertente assistencial, materializada no atendimento básico em nível primário à população local visando a elaboração do diagnóstico populacional de saúde, o que representou um grande avanço na contribuição social do projeto para a comunidade visitada, além de uma experiência adicional e inédita para os alunos da FMUSP. As realizações nesta nova fase começaram com Cajati SP e Eldorado-SP (Vale do Ribeira), em 1998 e 1999 respectivamente. A partir de 2000 , sob a coordenação do Prof. Carlos Corbett, também do departamento de patologia, o projeto passou a atuar fora do estado de São Paulo e no sentido de garantir a sustentabilidade e continuidade das ações, foram feitas parcerias com universidades locais, assim desenvolveram-se as expedições de Monte Negro-RO em 2000 e Buriticupu - MA em 2001.

\section{Fase 3 - Maturação e multidisciplinaridade}

Durante os três anos seguintes, com suas expedições para Serra dos Aymorés - MG em 2002; Presidente Epitácio-SP em 2003; e Teotônio Vilela e São José da Tapera-AL em 2004, o projeto tomou corpo e amadureceu sua forma de operacionalização e desenvolvimento de atividades. O crescimento dos então denominados Programa de Saúde da Família e Programa de Agentes Comunitários de Saúde, passou a garantir maior acesso da população à atenção básica e gerar maior demanda por atendimentos especializados. A Bandeira se adaptou a isto ampliando o leque de especialidades médicas envolvidas no projeto e incluindo também pela primeira vez uma equipe de alunos e professores do curso de fisioterapia da USP, o que se tornaria o embrião de uma nova e produtiva alteração conceitual do projeto.

A análise periódica dos dados coletados nas expedições mostrou que a atuação da equipe médica tinha efeitos importantes, mas que também tinha algumas limitações, especialmente resultantes do conceito mais restrito de saúde adotado pela Bandeira até então. Nesta nova fase, novamente sob a supervisão do Professor Paulo Saldiva e do Dr. Luiz Fernando Silva foi oficialmente adotada pela Bandeira a definição de saúde da Organização 
Mundial da Saúde, anteriormente citada, o que resultou na progressiva incorporação de outras áreas do conhecimento iniciando-se com a nutrição na expedição a João Câmara, Jandaíra e Bento Fernandes - RN em 2005, culminando com a inclusão da odontologia, psicologia, agronomia, além da engenharia civil e ambiental na expedição para Machadinho D'Oeste - RO em 2006; e a inclusão do jornalismo e áudio visual na expedição de 2007 para Penalva - MA4.

\section{Fase 4 - A interdisciplinaridade e a continuidade}

A inclusão de novas áreas abriu também novos horizontes para o projeto. No início estas áreas do conhecimento atuavam no mesmo local, mas com suas atividades específicas formando um projeto com múltiplas atividades não finamente alinhadas entre si. Com o passar do tempo os próprios alunos de cada área passaram a conhecer melhor as atividades $\mathrm{e}$ atuação de outras áreas do conhecimento o que permitiu o avanço no sentido de se desenvolverem atividades interdisciplinares nos diferentes pontos de atendimento, e assim foram as expedições de Itaobim - MG em 2008; Ivinhema - MS em 2009; Inhambupe - BA em 2010 e Belterra - PA em 2011. Para potencializar esta interação diversas atividades foram criadas reuniões interdisciplinares e a partir destas projetos conjuntos como interação engenharia e fisioterapia para construção de cadeiras de rodas; odontologia, fonoaudiologia e psicologia na atenção à saúde bucal, com apoio da fisioterapia para a ergonomia dos participantes; agronomia, medicina e fisioterapia para atuação nas comunidades rurais afastadas; visitas domiciliares com até 8 áreas envolvidas dependendo das necessidades dos casos, entre outros.

Além disso, foram estabelecidas em conjunto diversas atividades buscando garantir a sustentabilidade e continuidade das atividades do projeto através do reforço na elaboração de projetos estruturais para o município nas áreas de saúde e saneamento, formação e capacitação de multiplicadores e profissionais locais de saúde, estímulo ao desenvolvimento de projetos de extensão semelhantes pelas universidades parceiras, uso de ferramentas de comunicação à distância para acompanhamento de indicadores e apoio à cidade.

\section{ESTRATÉGIAS DE ATUAÇÃO}

O projeto é desenvolvido em três etapas fundamentais a saber:

1) Preparação - Neste período que dura em média oito meses os municípios candidatos são avaliados em termo necessidades, potenciais de atuação, e indicadores pré-estabelecidos como índice de desenvolvimento humano (IDH), população, densidade demográfica e cobertura da estratégia de saúde da família maior que $75 \%$. No período são realizadas em média quatro visitas de preparação para levantamento de demandas, estabelecimento de parcerias e organização das diferentes atividades junto aos gestores, instituições da sociedade civil organizada e comunidade local Ao mesmo tempo a equipe em São Paulo prepara as atividades (científicas, educativas e assistenciais) específicas de cada área do conhecimento e através de reuniões interdisciplinares discute as abordagens conjuntas. Nesta fase são também coletadas todas as informações necessárias para o bom desenvolvimento da expedição (informações gerais de saneamento que estão diretamente vinculadas aos aspectos de saúde, identificação de uma rede de multiplicadores e referências obtidas na própria sociedade através de entrevistas seqüenciais, entre outros). Nesta fase também são feitos os processos de seleção e treinamento dos participantes, alunos e profissionais que passam por cursos preparatórios, atividades gerais relacionadas não apenas a aspectos técnicos, mas também geográficos e culturais, atividades pós-seleção com treinamento de ações, simulações e estratégias. Nesta fase são ainda levantadas as demandas que têm potencial para desenvolvimento de trabalhos científicos com impacto local. Os projetos são redigidos e encaminhados para os respectivos comitês de ética para que possam ser desenvolvidos na fase de expedição

2) Expedição - Com duração aproximada de 10 dias e contando com uma equipe da ordem de duas centenas de alunos e profissionais, é nela que são desenvolvidas as principais atividades assistenciais, educativas e científicas. Elas ocorrem em até 8 pontos do município simultaneamente, três deles com grande integração, que são os chamados "Postos de Atendimento" que concentram equipes de medicina, odontologia, fisioterapia, fonoaudiologia, psicologia e nutrição. Nestes postos os indivíduos passam por estratégias de coleta de informações sociais e epidemiológicas, exames de rastreamento, e a seguir atendimento e orientação em saúde. Além das atividades específicas de cada área, diversas atividades são realizadas em conjunto, em caráter interdisicplinar, como por exemplo as visitas domiciliares a pacientes restritos ao leito ou com dificuldades de locomoção, atividades com comunidades ribeirinhas / agrícolas, e atividades com crianças em escolas. Estas atividades além de favorecem a integração entre as diferentes áreas 
do conhecimento, possibilita maior reconhecimento dos seus limites e potencialidades de ação. Embora a estrutura geral seja semelhante todos os anos, as características das atividades desenvolvidas pode variar consideravelmente de acordo com as demandas observadas em cada município.

3) Seguimento - Após a expedição o projeto entra em sua terceira fase, de duração média de 8 meses, que consiste no seguimento e desenvolvimento de estratégias de continuidade. Estas estratégias podem ser divididas em dois grupos: individuais e coletivas. A realização e acompanhamento destas estratégias é feita presencialmente (através de 4 a 6 visitas de seguimento) ou à distância.

a) Estratégias individuais - são aquelas referentes à relação da Bandeira Científica com os indivíduos diretamente atendidos por ela e incluem o envio de resultados de exames, doação de óculos e próteses quando necessários, encaminhamentos para serviços de referência dentro do Sistema Único de Saúde para prosseguimento ou acompanhamento do atendimento e disponibilização de informações em saúde através de materiais específicos ou à distância (utilizando-se ferramentas de internet e celular, quando disponíveis);

b) Estratégias coletivas - são aquela que impactam a população como um todo através de ações educativas, assistenciais ou de políticas públicas ou através da replicação do projeto localmente. Estão incluídas nestas estratégias os cursos de capacitação de profissionais de saúde ou lideranças locais enfocando os resultados obtidos na expedição; geração de relatórios e projetos técnicos com sugestões para organização estrutural e operacional da atenção à saúde e saneamento, proposição de estratégias preventivas, estímulo à parcerias com universidades locais para replicação do projeto e apresentação de resultados científicos de interesse coletivo.

\section{RESULTADOS}

Um projeto abrangente como a Bandeira Científica pode ter seus resultados avaliados sob diferentes prismas. Destacaremos alguns aspectos que consideramos relevantes ao avaliar o impacto obtido pelo projeto ao longo das suas últimas 14 expedições.

\section{Equipe}

Ao longo do tempo, com a inclusão de atividades assistenciais, ampliação das atividades de educação e pesquisa e a inclusão de novas áreas a Bandeira Científica observou um crescimento considerável de sua equipe tendo atingido nos últimos três anos um equilíbrio (Figura 1). Esta equipe conta fundamentalmente com alunos de graduação e profissionais e docentes que supervisionam diretamente as atividades em campo. O projeto conta hoje com a participação de 8 unidades da USP, respondendo por diferentes abordagens no projeto e também com diferentes proporções em termos de composição da equipe (Figura 2).

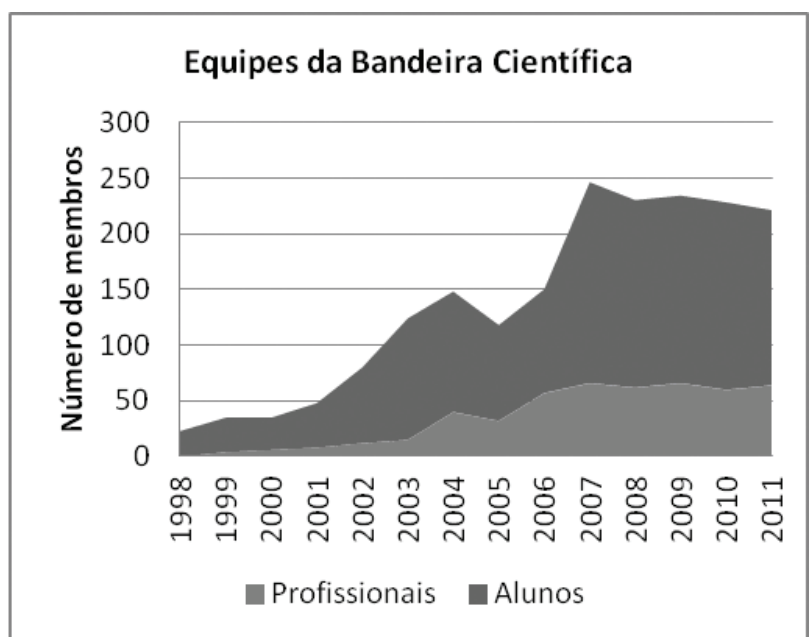

Figura 1. Evolução histórica da equipe da Bandeira Científica incluindo alunos e profissionais

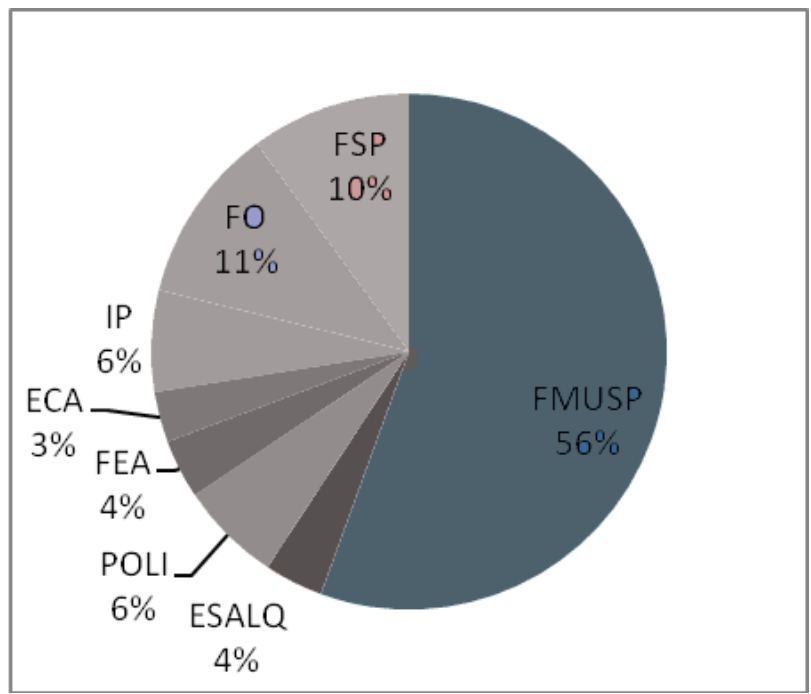

Figura 2 - Proporção média da composição da equipe em relação às unidades da USP

Nos últimos 14 anos o projeto contou com 1428 alunos, sendo 1152 da Universidade de São Paulo e 276 de instituições parceiras. O número de inscritos anualmente também é crescente e a razão média atual é de três candidatos para cada vaga disponibilizada evidenciando o interesse do corpo discente no projeto.

É interessante observar que a equipe não 
apenas impacta o projeto em relação ao número e qualidade das atividades, mas também é impactada por eles. Resultados preliminares de um estudo qualitativo em andamento mostram que para mias de $70 \%$ dos ex-bandeirantes da área médica, o projeto mudou sua forma de encarar a relação médicopaciente e sua visão da realidade do sistema de saúde do país.

\section{A população impactada}

Dados do número de pessoas atingidas diretamente pelo projeto e o número total de atividades pode ser visto na Tabela 1. Observamos que com o passar do tempo, especialmente a partir de 2005 o número de atividades individuais é significativamente maior do que o de pessoas diretamente impactadas, fato que passou a ocorrer preferencialmente após a inclusão de novas áreas do projeto uma vez que o mesmo indivíduo passava a participação em mais de uma atividade individual do projeto, em cada momento com uma área diferente através de orientações ou encaminhamentos.

Ainda em relação às atividades, observamos um aumento progressivo do número de exames realizados (Tabela 1 ) reflexo direto do aumento progressivo das necessidades locais de contenção de demandas reprimidas relacionadas a exames adicionais. Neste processo foram incluídos exames de sangue, eletrocardiogramas, ultrassonografias entre outros. A relevância do atendimento especializado na área médica e odontológica pode também ser observado pelo número de óculos doados à população e próteses produzidas.

Além da população impactada diretamente, atividades educativas para diferentes profissionais de educação e saúde atingem em média 100 pessoas diretamente e cerca de 1000 pessoas de forma indireta considerando o papel multiplicador destes profissionais capacitados. As principais atividades incluem: a) oficinas de capacitação para professores e agentes de saúde; b) ciclos de atualização em temas relevantes para médicos e agentes de saúde; e c) discussão de casos interessantes com as equipes da estratégia de saúde da família, geralmente incluindo casos da própria comunidade identificados durante a expedição.

Atividades educativas para a população geral na forma de palestras ou cursos básicos de suporte básico de vida e noções gerais de higiene e saúde já atingiram mais de 2500 pessoas diretamente durante a fase moderna da Bandeira Científica.

Atuando neste formato a Bandeira Científica já atendeu, nestes últimos 14 anos quase 50.000 pessoas em 17 municípios de quatro regiões do país.

TABELA 1 - Evolução anual dos indivíduos atendidos e atividades desenvolvidas na Bandeira Científica

\begin{tabular}{l|c|c|c|c|c|c|c|c|c|c|c|c|c|c}
\hline & $\mathbf{9 8}$ & $\mathbf{9 9}$ & $\mathbf{0 0}$ & $\mathbf{0 1}$ & $\mathbf{0 2}$ & $\mathbf{0 3}$ & $\mathbf{0 4}$ & $\mathbf{0 5}$ & $\mathbf{0 6}$ & $\mathbf{0 7}$ & $\mathbf{0 8}$ & $\mathbf{0 9}$ & $\mathbf{1 0 \#}$ & $\mathbf{1 1}^{*}$ \\
\hline Indivíduos & 293 & 700 & 1217 & 2322 & 1522 & 1935 & 4250 & 3690 & 4425 & 5210 & 5520 & 5220 & 5354 & 4980 \\
\hline Atividades & 320 & 939 & 2133 & 4040 & 3437 & 2662 & 6074 & 5984 & 7058 & 7120 & 7692 & 8695 & 8268 & 7650 \\
\hline Óculos & - & - & - & - & - & - & 242 & 446 & 725 & 631 & 544 & 610 & 705 & 600 \\
\hline Exames & 256 & 159 & 345 & 476 & 269 & 155 & 416 & 670 & 1022 & 649 & 1825 & 1723 & 1823 & 1513 \\
\hline Próteses & - & - & - & - & - & - & - & - & 24 & 24 & 24 & 24 & 0 & 30 \\
\hline
\end{tabular}

* Dados parciais em fase de consolidação; \# em 2010 a equipe de prótese não pode participar da Bandeira Científica

\section{Continuidade e Replicação}

Um dos objetivos centrais da Bandeira Científica é que suas atividades e a consolidação das informações obtidas impactem para a saúde pública local através da implementação de projetos, readequação estrutural e melhoria da qualidade de vida da população. Neste sentido alguns exemplos chamam a atenção:

Em Buriticupu-MA a parceria da Bandeira Científica com o poder público e a universidade local possibilitou a ampliação das atividades no núcleo de medicina tropical da cidade (coordenado pela Universidade Federal do Maranhão) e a reforma do Hospital Municipal local com base em projeto desenvolvido em parceria com a Bandeira.

Após a constatação, durante a expedição, de que a prevalência de doenças psiquiátricas era significativamente maior do que aquela encontrada no restante do país, foi preparado, a pedido do município, um relatório técnico detalhado sobre estes aspectos para servir como justificativa para a implantação 
de um Centro de Apoio Psico Social (programa do ministério da saúde) no município, apesar de ter uma população total menor que o preconizado. $O$ centro foi aprovado para implantação no ano seguinte.

Em 2011 a capacitação de lideranças locais para a construção de fossas sépticas e composteiras incluiu cerca de 15 lideranças que poderão replicar estes procedimentos de baixo custo melhorando as condições locais de saneamento.

Além deste processo de construção pensando na saúde coletiva, há também um acompanhamento direto da continuidade individual dos casos identificados na Bandeira Científica. Neste contexto, anualmente todos os resultados de exames são enviados por escrito ao paciente a à unidade de saúde onde o mesmo é cadastrado, são entregues as próteses e óculos (já são mais de 100 próteses e de 4000 óculos doados) e um acompanhamento direto de inclusão dos pacientes no sistema de saúde através de avaliação do percentual destes pacientes que teve o encaminhamento efetivado (no sistema de saúde local ou no de referência, dependendo do caso) após a expedição. Na Tabela 2, apresentamos os valores médios de efetivação dos encaminhamentos dos pacientes atendidos no projeto. Estes dados médios variam de acordo com particularidades dos estados e municípios atendidos. Em algumas expedições dificuldades na estrutura estadual de referência impossibilitaram o encaminhamento de todos os pacientes no período avaliado pela Bandeira Científica, motivo pelo qual não se observa, no período avaliado, a obtenção de taxas de $100 \%$ de encaminhamentos efetivados.

As crescentes parcerias da Bandeira Científica com instituições locais permitiram não apenas a troca de informações e conhecimentos de particularidades regionais por alunos e profissionais, mas também possibilitou a replicação do projeto por outras universidades. O exemplo mais recente deste processo foi a criação do Projeto UFMS Sem Fronteiras, pela Universidade Federal do Mato Grosso do Sul, após a experiência de parceria com a Bandeira Científica em 2009. Nos últimos dois anos o UFMS sem fronteiras realizou expedições para municípios no próprio estado nos mesmos moldes interdisciplinares da Bandeira Científica.

Tabela 2. Percentual de encaminhamentos efetivados ao longo dos meses de seguimento do Projeto Bandeira Científica - média de 13 anos*

\begin{tabular}{l|c|c|c|c|c|c|c|c}
\hline & $1^{\circ}$ mês & $2^{\circ}$ mês & $3^{\circ}$ mês & $4^{\circ}$ mês & $5^{\circ}$ mês & $6^{\circ}$ mês & $7^{\circ}$ mês & $8^{\circ}$ mês \\
\hline \% efetivação & 10 & 25 & 60 & 75 & 95 & 98 & 99 & 99 \\
\hline
\end{tabular}

* - No primeiro ano de reativação do projeto não houve atividade assistencial

\section{RESULTADOS CIENTÍFICOS}

Anualmente, resultados detalhados dos inquéritos epidemiológicos e sociais, bem como das diferentes atividades assistenciais, são analisados sobre o prisma científico, buscando sempre a elaboração de perguntas e a busca de conclusões relevantes para a comunidade local ${ }^{5-7}$.

Desta forma, ao longo dos últimos 14 anos os dados da Bandeira Científica já possibilitaram a realização de diversos trabalhos e atividades de iniciação científica conforme apresentado na Tabela 3

Tabela 3. Indicadores científicos e de divulgação da ciência alcançados pelo Projeto Bandeira Científica nos últimos 14 anos

Indicadores científicos e de divulgação da ciência

\begin{tabular}{l|c|l|c}
\hline Trabalhos de Conclusão de Curso & 22 & Artigos publicados & 14 \\
\hline Projetos de Iniciação Científica & 29 & Trabalhos em congressos nacionais & 32 \\
\hline Teses de Mestrado & 2 & Trabalhos em congressos internacionais & 19 \\
\hline Teses de Doutoramento & 2 & Relatórios e documentos estruturados & 19 \\
\hline & & Prêmios científicos & 4 \\
\hline
\end{tabular}

\section{Repercussão}

Outro aspecto importante é a repercussão extra-universitária da Bandeira Científica como projeto de extensão. Ao longo dos últimos 14 anos, a Bandeira Científica vem tendo grande exposição em diferentes mídias (Tabela 4), destacando-se uma edição completa do programa Globo Universidade em 2010(8). Além disso, o projeto já foi reconhecido com 
seis prêmios sociais incluindo Prêmio Saúde Brasil do Instituto Ethos e o Prêmio Cidadania Sem Fronteiras - Edição Nacional - do Instituto de Cidadania Brasil e Ministério da Ciência e Tecnologia ${ }^{9}$.

Tabela 4. Repercussão das atividades da Bandeira Científica na mídia nos últimos 14 anos

\begin{tabular}{l|c}
\hline \multicolumn{2}{c}{ Repercussão na Mídia } \\
\hline Notícias na mídia impressa & 72 \\
\hline Notícias na mídia eletrônica & 245 \\
\hline $\begin{array}{l}\text { Programas de televisão - } \\
\text { matérias }\end{array}$ & 21 \\
\hline $\begin{array}{l}\text { Programas de televisão - } \\
\text { temáticos }\end{array}$ & \multicolumn{1}{c}{4} \\
\hline Prêmios honoríficos & $\begin{array}{l}\text { 1. Comenda } \\
\text { Machadinho }\end{array}$ \\
\hline Prêmios sociais & $\begin{array}{l}\text { 5 - Saúde Brasil (2001 } \\
\text { e 2004), Cidadania } \\
\text { Sem Fronteiras (2009), } \\
\text { Top Social (2011), Lupa } \\
\text { de Ouro (2011). }\end{array}$ \\
\hline
\end{tabular}

\section{CONSIDERAÇÕES, ANÁLISE CRÍTICA E PERSPECTIVAS}

Considerando os dados apresentados, a evolução apresentada pela Bandeira Científica nos últimos anos ilustra um esforço que foi além da idéia de simples retomada de um projeto histórico da FMUSP. Ao longo do tempo equipe envolvida buscou trazer o enfoque assistencial, o educativo e o científico, equilibrando este triplo sustentáculo da Universidade, se esforçando também para atuar na orientação dos gestores para a formação de políticas públicas de saúde (avaliação e orientação sobre o modelo de organização de saúde local). Estes enfoques têm sido anualmente expandidos e melhorados através de reuniões anuais de apresentação de dados e discussão de estratégias e resultados; da ampliação da interdisciplinaridade do projeto, e desenhando diferentes estratégias de continuidade.

Não é pretensão da equipe da Bandeira a resolução todos os problemas, mas sim, a avaliação das condições de saúde e sugestão das possibilidades de atuação de longo prazo para um processo de transformação gradual envolvendo o poder público nas diversas esferas, universidades e representações comunitárias. Assim, as atividades realizadas em suas diversas fases servem como ponto de partida e sensibilização, tanto da população como das diversas entidades envolvidas, além de fornecer informações relevantes para o planejamento estratégico e definição de prioridades. E é justamente com este argumento que a cada ano a Bandeira Científica desenvolve atividades em regiões diferentes, entendendo que ela é apenas uma parte de um processo que inclui os gestores públicos locais e as universidades parceiras que continuarão desenvolvendo atividades na região.

Tudo isso deve ser feito sem esquecer o caráter de projeto acadêmico da Bandeira Científica e neste sentido ela tem disponibilizado aos alunos da USP uma experiência única, pois coloca esses jovens universitários em contato com a população de municípios distantes do Brasil, com realidades particulares, em geral precárias, e organização política e social também diversas. A vivência alcançada está além de contato com conjuntura social, mas passa pela reflexão sobre a cidadania.

Ao contrário de dados frios apresentados em salas de aula, da realidade de indicadores de saúde dos livros e artigos e da teoria da organização do Sistema Único de Saúde no Brasil, e das dificilmente abstraídas, o aluno é colocado no âmago da situação e, naquele período, passa a fazer parte dela. Neste processo ele passa a conviver lado a lado com as condições reais de vida e de assistência à saúde em nosso país, e se depara com o desafio de trabalhar em situações com escassos recursos complementares para auxiliar os diagnósticos e conhecer a realidade do atendimento em saúde vigente na maior parte dos municípios brasileiros. Certamente peculiaridades e dificuldades na atenção ao sistema de saúde são condições encontradas também periferia das grandes cidades e diferentes unidades da USP têm projetos atuando nestas comunidades. Entendemos porém que a vivência destas condições em comunidades distantes, onde não é possível simplesmente "voltar para casa" à noite, gera uma imersão tal que demandará do indivíduo, após seu retorno, uma intensa reflexão a respeito de sua posição e seu papel na sociedade.

A realidade hospitalar, por vezes demasiado precária destas regiões, e a observação das condições reais de vida permitem valorizar a necessidade de uma boa anamnese e exame clínico, aprendizado que é trazido na bagagem após o retorno da expedição, resultando em melhor abordagem clínica com redução do apelo desnecessário a exames complementares. Este trabalho mostra também a necessidade de cooperação multiprofissional e intersetorial e a importância de outros instrumentos, entre os quais destacamos a epidemiologia, para a compreensão dos problemas coletivos de saúde. O aluno começa assim a perceber que há diferença entre enxergar exclusivamente a saúde individual do paciente à sua frente, e a saúde populacional, como sistema que tem por objetivo suprir as necessidades de toda a população dentro dos preceitos constitucionais de 
igualdade. Consideramos que o conhecimento destes dois pontos de vista, que não são antagônicos, mas em sua complementaridade possuem diferenças importantes, é crucial para que eles, futuros profissionais, possam entender e participar da promoção de saúde tendo em mente não apenas uma parte desta visão, mas sim a complementaridade dela.

No desejo de melhorias imediatas, os dados epidemiológicos, derivados da análise das informações coletadas, são uma arma para consubstanciar as propostas de mudança e sustentabilidade do atendimento local numa perspectiva futura. A construção associada de um banco de informações permite o acesso a diversos outros cruzamentos e análises que podem servir para delinear ações, pesquisar necessidades e avaliar efeitos de determinadas atuações ou mudanças. Espera-se com isso multiplicar o trabalho científico, epidemiológico e clínico e, simultaneamente, sensibilizar alunos a considerarem novas questões sobre sua identidade profissional e de cidadão brasileiro.

A crescente procura dos acadêmicos pela Bandeira Científica mostra que está no rumo certo, não apenas em termos acadêmicos, mas em termos de sociedade e cidadania e torna evidente o crescente interesse dos alunos e sua consciência sobre a importância de se vivenciar a diversidade de realidades do país.

A USP tem funções e obrigações que vão além do ensino e capacitação técnica de seus alunos como profissionais isolados, devendo aliar a isto a formação de cidadãos conscientes da realidade do país e da população que arca com esta formação, para que então possam desenvolver suas atividades profissionais com responsabilidade profissional e social, integrando-se a novas realidades e formas de atuação.

A Bandeira Científica ainda tem grandes desafios pela frente incluindo potencializar a interdisciplinaridade e as atividades conjuntas, ampliar suas ferramentas de continuidade, especialmente usando tecnologias de comunicação à distância, já em implantação no projeto, mas que podem ser ampliadas. Existe ainda uma proposta ainda em discussão de retorno a um município atendido há cerca de 5 anos pelo projeto para uma comparação de realidades e desenvolvimento de pesquisas qualitativas do papel da Bandeira nisto. Em que pese o fato de que diversos fatores podem ter alterado as condições do município, tentar investigar se a Bandeira Científica foi, de alguma forma, um fator reconhecido como tal é ao mesmo tempo desafiador e estimulante para um projeto que vem buscando se adaptar às mudanças de realidade do país e aprimorar constantemente suas estratégias de ação.

Agradecimentos: Pró-Reitoria de Cultura e Extensão da USP. Diretorias das Unidades Participantes. Comissões de Cultura e Extensão da Unidades Participantes. Hospital das Clínicas da FMUSP e clínicas envolvidas (clínica geral, ginecologia, oftalmologia, otorrinolaringologia, dermatologia. Empresas apoiadoras: Grupo Sanofi, Fundação Faculdade de Medicina, Ministério da Defesa - Governo Federal, Força Aérea Brasileira, Exército Brasileiro, Instituto Vivo, Miguel Giannini Óculos, Grupo Essilor, Mowa, Finnet, Colgate, Cardioequipo.

\section{REFERÊNCIAS}

1. WHO. Definition of health [cited 2012 Feb 2]. Available from: https://apps.who.int/aboutwho/en/definition. html.

2. Ferreira E. A primeira bandeira científica. J FFM. 2010;9:11.

3. Marques F. Luiz rey, uma vida nada monótona. Rev Manguinhos. 2007:13.

4. Científica B. Relatórios do projeto bandeira científica. São Paulo: Universidade de São Paulo; 2001-2010. Disponível em: http://www.Bandeiracientifica.com.br.

5. da Silva LF, Saldiva SR, Saldiva PH, Dolhnikoff M. Impaired lung function in individuals chronically exposed to biomass combustion. Environ Res. 112:111-117.
6. Goldbaum M, Ribeiro RB. Prevalência de toxoplasmose, leishmaniose, doença de chagas e enteroparasitoses em voluntários da população de cajati - São Paulo. Rev Med. 1999;78:498-451.

7. El Khouri M, Duarte LS, Ribeiro RB, da Silva LF, Camargo LM, dos Santos VA, Burattini MN, Corbett CE. Seroprevalence of hepatitis $b$ virus and hepatitis $c$ virus in monte negro in the brazilian western amazon region. Clinics (Sao Paulo). 2005;60:29-36.

8. Globo RGdT. Projeto bandeira científica. 2010 [citado em 20 fev. 2012]. Disponível em: http://redeglobo.globo.com/globouniversidade/videos/t/edicoes/v/projetobandeira-cientifica/1525215/.

9. MCT/IC IC. Prêmio cidadania sem fronteiras. 2009. 